\title{
Video Article \\ Quantifying the Frequency of Tumor-propagating Cells Using Limiting Dilution Cell Transplantation in Syngeneic Zebrafish
}

\author{
Jessica S. Blackburn ${ }^{1}$, Sali Liu ${ }^{1}$, David M. Langenau ${ }^{2}$ \\ ${ }^{1}$ Department of Molecular Pathology, Massachusetts General Hospital, Harvard Medical School \\ ${ }^{2}$ Department of Molecular Pathology, Massachusetts General Hospital Cancer Center, Harvard Stem Cell Institute
}

Correspondence to: David M. Langenau at dlangenau@partners.org

URL: https://www.jove.com/video/2790

DOI: doi:10.3791/2790

Keywords: Developmental Biology, Issue 53, cancer stem cell, T-cell acute lymphoblastic leukemia, microinjection, fluorescence, self-renewal

Date Published: 7/14/2011

Citation: Blackburn, J.S., Liu, S., Langenau, D.M. Quantifying the Frequency of Tumor-propagating Cells Using Limiting Dilution Cell Transplantation in Syngeneic Zebrafish. J. Vis. Exp. (53), e2790, doi:10.3791/2790 (2011).

\section{Abstract}

Self-renewing cancer cells are the only cell types within a tumor that have an unlimited ability to promote tumor growth, and are thus known as tumor-propagating cells, or tumor-initiating cells. It is thought that targeting these self-renewing cells for destruction will block tumor progression and stop relapse, greatly improving patient prognosis ${ }^{1}$. The most common way to determine the frequency of self-renewing cells within a tumor is a limiting dilution cell transplantation assay, in which tumor cells are transplanted into recipient animals at increasing doses; the proportion of animals that develop tumors is used the calculate the number of self-renewing cells within the original tumor sample ${ }^{2,3}$. Ideally, a large number of animals would be used in each limiting dilution experiment to accurately determine the frequency of tumor-propagating cells. However, large scale experiments involving mice are costly, and most limiting dilution assays use only 10-15 mice per experiment.

Zebrafish have gained prominence as a cancer model, in large part due to their ease of genetic manipulation and the economy by which large scale experiments can be performed. Additionally, the cancer types modeled in zebrafish have been found to closely mimic their counterpart human disease ${ }^{4}$. While it is possible to transplant tumor cells from one fish to another by sub-lethal irradiation of recipient animals, the regeneration of the immune system after 21 days often causes tumor regression ${ }^{5}$. The recent creation of syngeneic zebrafish has greatly facilitated tumor transplantation studies ${ }^{6-8}$. Because these animals are genetically identical, transplanted tumor cells engraft robustly into recipient fish, and tumor growth can be monitored over long periods of time. Syngeneic zebrafish are ideal for limiting dilution transplantation assays in that tumor cells do not have to adapt to growth in a foreign microenvironment, which may underestimate self-renewing cell frequency ${ }^{9}$ ${ }_{10}$. Additionally, one-cell transplants have been successfully completed using syngeneic zebrafish ${ }^{8}$ and several hundred animals can be easily and economically transplanted at one time, both of which serve to provide a more accurate estimate of self-renewing cell frequency.

Here, a method is presented for creating primary, fluorescently-labeled T-cell acute lymphoblastic leukemia (T-ALL) in syngeneic zebrafish, and transplanting these tumors at limiting dilution into adult fish to determine self-renewing cell frequency. While leukemia is provided as an example, this protocol is suitable to determine the frequency of tumor-propagating cells using any cancer model in the zebrafish.

\section{Video Link}

The video component of this article can be found at https://www.jove.com/video/2790/

\section{Protocol}

\section{DNA microinjection of zebrafish embryos}

1. Linearize rag2::CMyc and rag2::GFP ${ }^{11}$ DNA constructs by digesting $10 \mu \mathrm{g}$ of each plasmid with Notl at $37^{\circ} \mathrm{C}$ overnight.

2. Phenol:chloroform extract and precipitate the plasmids, and resuspend each in $20 \mu \mathrm{L} \mathrm{H}_{2} \mathrm{O}$.

3. Determine the concentration of the DNA by running a $1: 1,1: 5$ and $1: 10$ dilution of each digested plasmid on a $1 \%$ agarose gel with $20 \mu \mathrm{L}$, $10 \mu \mathrm{L}$ and $5 \mu \mathrm{L}$ of a high-range DNA ladder. This type of quantification is generally more accurate than a spectrometer reading.

4. Dilute the DNA to a final concentration of $60 \mathrm{ng} / \mu \mathrm{L}$ in $1 \mathrm{M} \mathrm{KCl}+0.5 \mathrm{X} \mathrm{TE}$ for injection into $\mathrm{CG} 1$-strain (or other syngeneic strain) zebrafish embryos using 30ng/ $\mu \mathrm{L}$ rag2::cMyc + 30ng/ $\mu \mathrm{L}$ rag2::GFP.

5. Injections should be performed as previously demonstrated ${ }^{12,13}$. Briefly, male and female zebrafish are set up in mating chambers the night before injection. On the day of injection, DNA is loaded into the injection needle, the pressure is adjusted so that a bubble with a 50 $\mu m$ diameter is expelled (30pg DNA), which is measured by a stage micrometer. Then, embryos are injected at the one-cell stage, with the DNA being injected directly into the cell, and not into the yolk. Survival of the injected CG1 embryos and larvae is generally poor, and only $5 \%$ of fish will successfully incorporate the transgene; thus, $>600$ embryos should be injected to ensure that some fish will develop leukemia.

6. Store the injected embryos at $28.5^{\circ} \mathrm{C}$, and remove the dead embryos after $24 \mathrm{hr}$. Animals are then transferred to large tanks at 5 days of life and raised as previously described ${ }^{14}$. 


\section{Screening for primary T-ALL in zebrafish larvae}

1. Approximately 28 days after injection, anesthetize larval zebrafish by adding $200 \mu \mathrm{L}$ of $4 \mathrm{ng} / \mathrm{m}$ Tricane-S to $25 \mathrm{~mL}$ of fish system water in a petri dish.

2. Examine the larvae for development of fluorescently-labeled T-ALL by using an epifluorescence microscope at 20X magnification, using a 485/20 excitation wavelength and 530/25 emission filter to detect GFP fluorescence (Figure 1).

3. Separate tumor positive larvae from those that are negative. Negative larvae can be examined at a later time point, once tumors may have grown larger, to ensure that no T-ALL positive fish were missed.

4. Monitor T-ALL positive fish at least once at week using the epifluorescence microscope to track tumor growth.

\section{Isolation and purification of fluorescently-labeled primary leukemia cells}

1. When GFP-positive leukemia cells have overtaken $>50 \%$ of the animal, sacrifice the fish by adding $1 \mathrm{~mL}$ of $4 \mathrm{ng} / \mathrm{mL}$ Tricane-S in a petri dish containing $9 \mathrm{~mL}$ fish system water.

2. Place the fish in a new petri dish containing $1 \mathrm{~mL} 0.9 \mathrm{X}$ PBS with $5 \%$ Fetal Bovine Serum to buffer the cells. Macerate the fish with a razor blade, pipet the mixture to dissociate large cell clumps, then pass the cells through a $40 \mu \mathrm{m}$ mesh strainer into a $50 \mathrm{~mL}$ tube. It is not necessary to dissociate all tissue clumps into single cells.

3. Pipet $500 \mu \mathrm{L}$ of the cells to a $4 \mathrm{~mL}$ polystyrene tube. Add $1 \mu \mathrm{L} 1 \mathrm{mg} / \mathrm{mL}$ propidium iodide $(\mathrm{PI})$, which will label dead cells. Vortex, then keep the cells on ice.

4. Sacrifice a wild-type CG1 fish, macerate and strain in the same way as above to collect normal cells, which serve as a carrier for the tumor cells during transplant.Add $4 \mathrm{~mL} 5 \%$ FBS in $0.9 \times$ PBS to the $50 \mathrm{~mL}$ tube for a total volume of $5 \mathrm{~mL}$.

5. Count the normal cells, dilute to $3 \times 10^{5}$ cells $/ \mathrm{mL}$ in $5 \%$ FBS in $0.9 \times$ PBS, then pipet $100 \mu \mathrm{L} /$ well of a 96 -well plate

6. Using a Fluorescence Activated Cell Sorter (FACS), sort GFP positive, PI negative tumor cells (Figure 2A,B) into the 96-well plate containing blood cells at the following doses: 10 cells/well into 48 wells, 100 cells/well into 24 wells, 1000 cells/well into 12 wells, and 10,000 cells/well into 6 wells. Additionally, 10,000 cells should be sorted into a well without normal blood cells, and reanalyzed using FACS to assess the purity and viability of the sorted cells (Figure $2 \mathrm{C}$ ).

\section{Limiting dilution transplantation into adult zebrafish}

1. Pellet the cells by centrifuging the 96 -well plate at $2000 \mathrm{xg}$ for 10 minutes.

2. Remove $95 \mu \mathrm{L}$ of the supernatant, and resuspend the cells in the remaining $5 \mu \mathrm{L}$.

3. Hold an adult (>60 days old) syngeneic zebrafish ventral side up, and inject the cell suspension into the peritoneal cavity, using a $26 \mathrm{G} / 2$ " micro-syringe. Zebrafish do not have to be anesthetized before transplant. Typically, 45 fish are transplanted with 10 leukemia cells, 20 are transplanted with 100 cells, 7 are transplanted with 1,000 cells and 5 fish are transplanted with 10,000 T-ALL cells.

\section{Analysis to determine leukemia-initiating cell frequency}

1. Approximately 28 days after transplant, examine the transplanted fish with an epifluoresence microscope using a $485 / 20$ excitation wavelength and 530/25 emission filter to detect GFP fluorescence. Record the number of positive fish per total number of fish injected.

2. Re-examine the fish every 14 days for up to 90 days and record the number of positive fish. When a tumor-positive fish become moribund sacrifice the animal by Tricane-S overdose.

3. Input the results into a table, as shown in Figure 3D. Upload the data into the web-based ELDA (Extreme Limiting Dilution Analysis) statistical software at http://bioinf.wehi.edu.au/software/elda/index.html ${ }^{15}$, which uses the frequency of tumor positive and negative animals at each transplant dose to determine the frequency of self-renewing leukemia cells within the primary T-ALL.

\section{Representative Results:}

DNA microinjection into embryos from syngeneic fish often results in a low survival rate of the injected embryos, with $<60 \%$ of embryos surviving for 24 hours. Additionally, survival of the injected syngeneic fish is generally poor during larval development, with often $<20 \%$ surviving for 28 days. It is therefore important to inject a large number of embryos in order to create transgenic fish that will develop T-ALL.

As an example, CG1 strain embryos were injected with rag2::cMyc+rag2::GFP. The transgenes co-segregate in the developing embryo, so that every cell that expresses GFP will also express cMyc. Larvae were screened for primary T-ALL after 28 days (Figure 1). Previous work has shown that approximately $5 \%$ of injected fish will have GFP-positive T-cells within their thymus (Figure 1), and $100 \%$ of these fish will develop leukemia as the T-cells become transformed ${ }^{11}$. While a small proportion of zebrafish may have a more advanced leukemia that is very easy to detect at day 28 , most will only have a GFP-positive thymus (Figure 1), so larvae should be examined individually under high magnification to ensure that all positive fish are selected. GFP-positive T-ALL cells will overtake $>50 \%$ of the animal between days $45-70$.

In the example provided, zebrafish were sacrificed at 65 days of life, and leukemia cells were isolated and FACS sorted for limiting dilution transplantation. Single cells that were propidium iodide negative and GFP-positive (Figure 2) were sorted into a 96-well plate. Reanalysis was done on 10,000 sorted cells in order to assess viability (ideally $>95 \%$ ) and purity (ideally $>92 \%$, Figure 2 ). Transplanted T-ALLs generally arise before 28 days, but some tumors may take more than 60 days to develop. In this example, at day 28, early stage tumors were seen as an area of GFP-positive cells near the injection site (Figure 3B), while some T-ALLs were more progressed, having expanded to fill the peritoneal cavity (Figure 3C). Data from limiting dilution cell transplantation assays from three different primary T-ALLs are shown in Figure 3D. For these experiments, over 220 animals were transplanted, which can be easily accomplished by one person within several hours. Data analysis using ELDA software showed that, on average, 1 in 135 T-ALL cells were self-renewing leukemia-propagating cells (Figure 3E). 


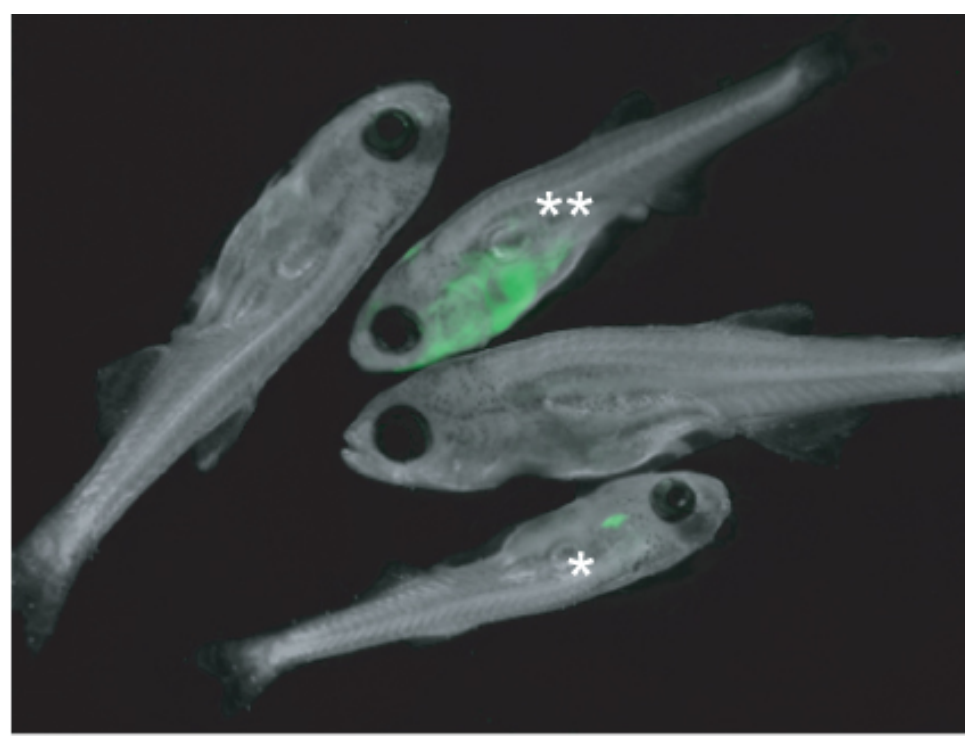

Figure 1. Zebrafish larvae injected at the one-cell stage with rag::-cMyc+rag::-eGFP were screened for primary leukemia growth 28 days postinjection. Fish $\left(^{*}\right)$ had GFP-positive T-cells within the thymus; this fish will develop T-ALL as the T-cells become transformed over time. This stage is very common at day 28 . One fish $\left(^{* *}\right)$ had an advanced T-ALL that has spread into the soft tissue. The remaining two fish are negative for tumor growth. The image was taken at $16 \mathrm{X}$ magnification.
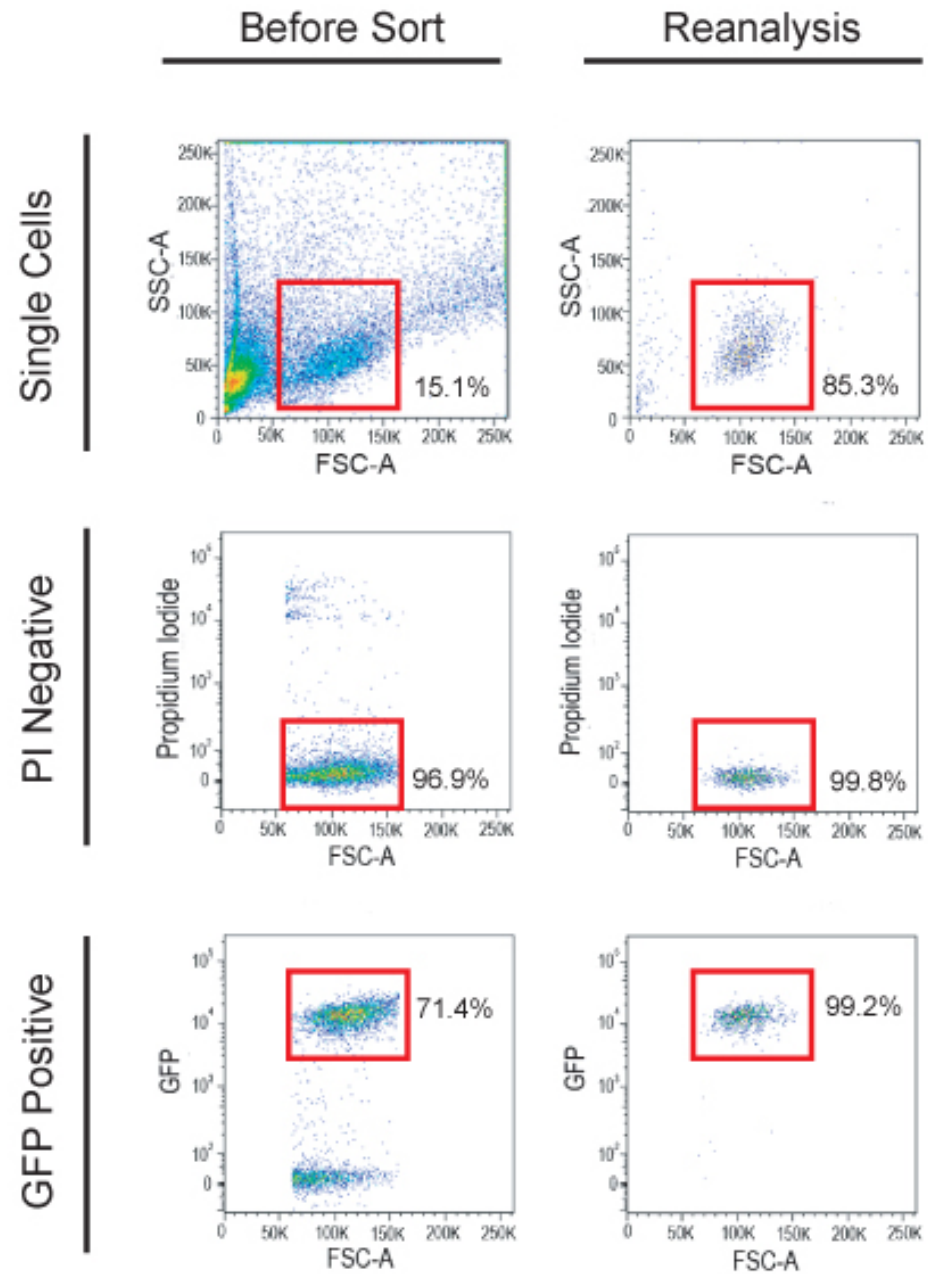

Figure 2. Fluorescently-labeled T-ALL cells were sorted from diseased animals and used in the limiting dilution cell transplantation assay. First, a gate was drawn to select single cells (upper left panel), then propidium iodide negative cells are selected (middle left panel). Finally, a gate was 
drawn to select only the GFP-positive leukemia cells for sorting (lower left panel). Sorted cells should be reanalyzed to assess viability and purity before transplant (right panels).

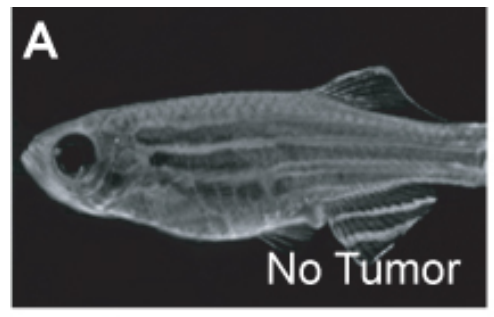

D
\begin{tabular}{|c|c|c|c|}
\hline Dose (Cells) & Transplanted & Engrafted & Group \\
\hline 10,000 & 5 & 5 & T-ALL-1 \\
\hline 1,000 & 7 & 7 & T-ALL-1 \\
\hline 100 & 21 & 6 & T-ALL-1 \\
\hline 10 & 40 & 4 & T-ALL-1 \\
\hline 10,000 & 5 & 5 & T-ALL-2 \\
\hline 1,000 & 7 & 7 & T-ALL-2 \\
\hline 100 & 21 & 11 & T-ALL-2 \\
\hline 10 & 42 & 9 & T-ALL-2 \\
\hline 10,000 & 5 & 5 & T-ALL-3 \\
\hline 1,000 & 7 & 7 & T-ALL-3 \\
\hline 100 & 20 & 11 & T-ALL-3 \\
\hline 10 & 42 & 6 & T-ALL-3 \\
\hline
\end{tabular}

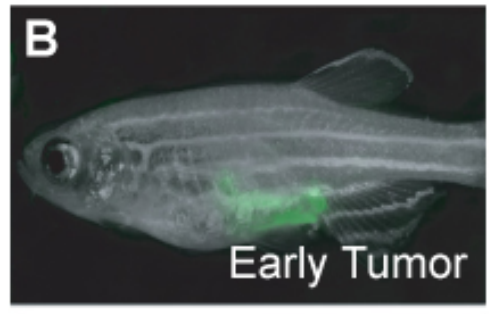

E
\begin{tabular}{|c|c|c|c|}
\hline \multicolumn{4}{|c|}{ Confidence Intervals } \\
\hline Group & Lower $95 \%$ & Estimate & Upper $95 \%$ \\
\hline T-ALL:1 & 378.6 & 210.1 & 116.7 \\
\hline T-ALL:2 & 146.8 & 91.3 & 56.8 \\
\hline T-ALL:3 & 170.2 & 103.5 & 63 \\
\hline
\end{tabular}

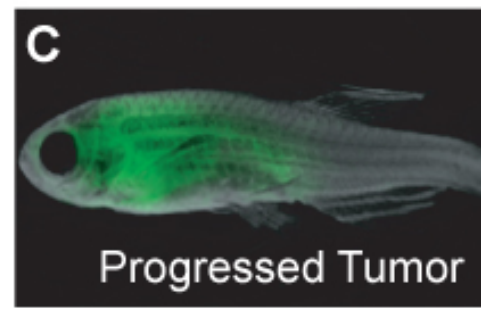

Figure 3. Zebrafish were examined for leukemia growth 28 days after transplant. Fish are either tumor negative (A), have a small tumor growing at the injection site $(B)$, or have a progressed leukemia (C). The images were taken at $7 X$ magnification. The total number of leukemia-positive fish per total number of fish transplanted at each dilution is recorded, as in (D). The data are input into the web-based ELDA program to calculate the number of self-renewing leukemia cells and the upper and lower $95 \%$ confidence intervals (E).

\section{Discussion}

A major strength of using zebrafish in cancer research is that large numbers of animals can be used at relatively low cost. This is especially important in limiting dilution cell transplantation assays, where the proportions of transplanted animals that develop tumors to the total number transplanted are used to determine tumor-initiating cell frequency. In the method presented here, over 70 transplant recipient animals are used per assay, providing an accurate estimate of tumor-propagating cell number. Both the number of animals used and the doses of tumor cells transplanted should be optimized for a given cancer model; for example, if initial experiments show tumor-initiating cells are rare, useful transplant doses may be $100,000,50,000,10,000$ and 1,000 cells per transplant.

Syngeneic zebrafish strains are useful in limiting dilution analysis. However, these strains are not yet commonly used in all laboratories, and some zebrafish cancer models exist only in allogenic fish. Limiting dilution cell transplantations can be performed in wild-type strains that have undergone immune ablation, although the self-renewing cell frequency may be calculated as 10-100 fold higher than if syngeneic zebrafish were used $^{8}$. It is important to note that greater doses of cells should be used for transplants into non-immune matched, irradiated recipients, as some tumor cells will not survive transplant into a foreign microenvironment. Additionally, many tumors will begin to regress after 21 days when the recipient animal's immune system recovers, so animals should be assessed for tumor growth every 7 days after transplant.

The methods presented here are suitable for use with any zebrafish cancer model, and have thus far been used to determine tumor-initiating cell frequency in both T-cell acute lymphoblastic leukemia ${ }^{8}$, and a solid tumor model, rhabdomyosarcoma ${ }^{16}$. These tumors were fluorescently labeled by co-injection of embryos with both an oncogene and fluorescent marker; because the DNA co-segregates, any cell expressing the oncogene will also express the fluorescent protein ${ }^{17}$. While fluorescence is recommended because it facilitates tracking of tumor growth without the need to sacrifice the animal and provides a straight-forward way to isolate tumor cells by FACS, it is possible to label tumor cells with antibodies to tumorspecific makers to facilitate their purification, though antibody staining in zebrafish may require optimization.

Finally, once the incidence of tumor-propagating cells has been determined for a given tumor type, it is possible to use these assays to identify the mechanisms that govern their cell frequency. For example, primary tumor cells can be treated with pathway specific inhibitors before limiting dilution transplant, or transplanted fish themselves may be dosed with drugs. Additionally, the genetics of the tumors themselves may be manipulated by injecting the one-cell stage fish with a gene of interest, so that the resulting primary tumors will over-express the gene. In these experiments, any effect on self-renewing cell frequency will be observed in the limiting dilution assays. 


\section{Disclosures}

No conflicts of interest declared.

\section{Acknowledgements}

Funding has been provided by NIH grants 5T32CA09216-26 (for J.S.B.), and K01 AR055619-01A1 and 3 K01 AR055619-03S1 (for D.M.L.), as well as by the Alex's Lemonade Stand Foundation, the Harvard Stem Cell Institute and the American Cancer Society.

\section{References}

1. Zhou, B.-B.S., et al. Tumour-initiating cells: challenges and opportunities for anticancer drug discovery. Nat Rev Drug Discov. 8, 806-823 (2009).

2. Dick, J.E., Bhatia, M., Gan, O., Kapp, U., \& Wang, J.C.Y. Assay of human stem cells by repopulation of NOD/SCID mice. Stem Cells. 15, 199-207 (1997).

3. Bonnefoixa, T., Bonnefoixa, P., Verdiela, P., \& Sotto, J.-J. Fitting limiting dilution experiments with generalized linear models results in a test of the single-hit Poisson assumption J Immunol Methods. 194, 113-119 (1996).

4. Stoletov, K., \& Klemke, R. Catch of the day: zebrafish as a human cancer model. Oncogene. 27, 4509-4520 (2008).

5. Taylor, A.M., \& Zon, L.I. Zebrafish Tumor Assays: The State of Transplantation. Zebrafish. 6, 339-346 (2009).

6. Mizgirev, I., \& Revskoy, S. Generation of clonal zebrafish lines and transplantable hepatic tumors. Nat Protocols. 5, 383-394 (2010).

7. Mizgirev, I.V., \& Revskoy, S.Y. Transplantable Tumor Lines Generated in Clonal Zebrafish. Cancer Research. 66, 3120-3125 (2006).

8. Smith, A.C.H., et al. High-throughput cell transplantation establishes that tumor-initiating cells are abundant in zebrafish T-cell acute lymphoblastic leukemia. Blood. 115, 3296-3303 (2010).

9. Kelly, P.N., Dakic, A., Adams, J.M., Nutt, S.L., \& Strasser, A. Tumor Growth Need Not Be Driven by Rare Cancer Stem Cells. Science. 317, 337 (2007).

10. Rosen, J.M., \& Jordan, C.T. The Increasing Complexity of the Cancer Stem Cell Paradigm. Science. 324, 1670-1673 (2009).

11. Langenau, D.M. et al. In vivo tracking of T cell development, ablation, and engraftment in transgenic zebrafish. Proceedings of the National Academy of Sciences of the United States of America. 101, 7369-7374 (2004).

12. Rosen, J.N., Sweeney, M.F., \& Mably, J.D. Microinjection of Zebrafish Embryos to Analyze Gene Function. J Vis Exp., e1115 (2009).

13. Yuan, S., \& Sun, Z. Microinjection of mRNA and Morpholino Antisense Oligonucleotides in Zebrafish Embryos. J Vis Exp., e1113 (2009).

14. Westerfield, M. The zebrafish book. A guide of the laboratory use of zebrafish (Danio rerio), Edn. 4. (University of Oregon Press, 2000).

15. Hu, Y., \& Smyth, G.K. ELDA: Extreme limiting dilution analysis for comparing depleted and enriched populations in stem cell and other assays. Journal of Immunological Methods. 347, 70-78 (2009).

16. Ignatius, M.S., et al. In vivo imaging identifies that myf5+ embryonal rhabdomyosarcoma-propagating cells are dynamically reorganized during tumor growth. under review (2010).

17. Langenau, D.M., et al. Co-injection strategies to modify radiation sensitivity and tumor initiation in transgenic Zebrafish. Oncogene. 27, 4242-4248 (2008). 